\title{
Particle charge in PK-4 dc discharge from ground-based and microgravity experiments
}

Cite as: Phys. Plasmas 26, 113703 (2019); https://doi.org/10.1063/1.5122861

Submitted: 03 August 2019. Accepted: 24 October 2019. Published Online: 08 November 2019

T. Antonova, S. A. Khrapak (D), M. Y. Pustylnik, M. Rubin-Zuzic, H. M. Thomas (D), A. M. Lipaev, A. D. Usachev, V. I. Molotkov, and M. H. Thoma (D)

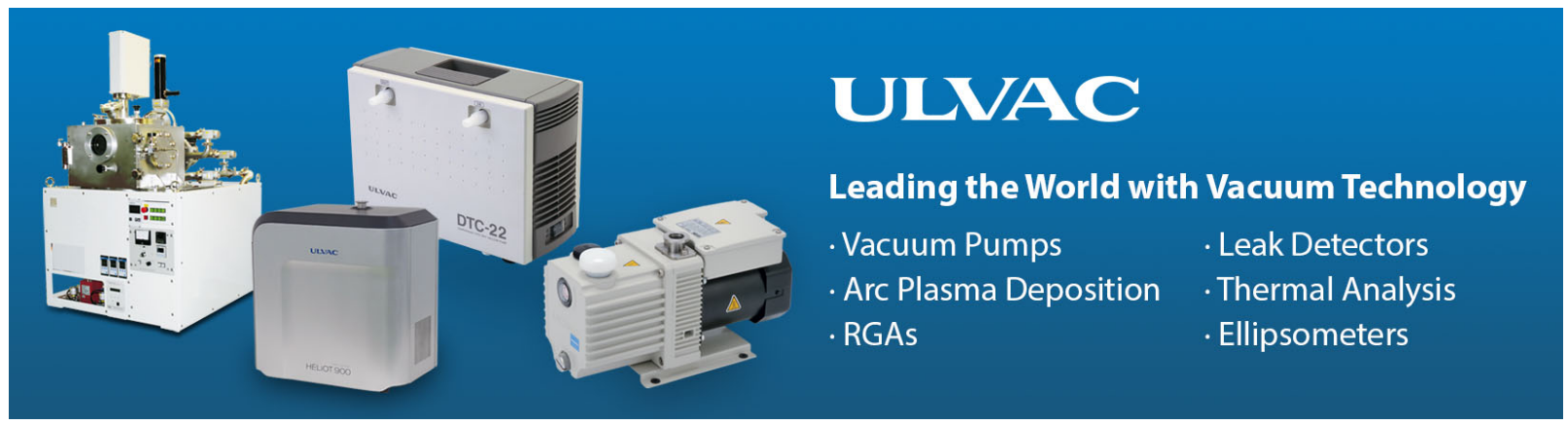




\title{
Particle charge in PK-4 dc discharge from ground-based and microgravity experiments
}

\author{
Cite as: Phys. Plasmas 26, 113703 (2019); doi: 10.1063/1.5122861 \\ Submitted: 3 August 2019 - Accepted: 24 October 2019 . \\ Published Online: 8 November 2019
}

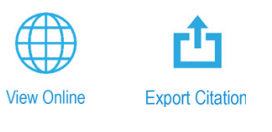
T. Antonova, ' S. A. Khrapak, ${ }^{1}$ (D) M. Y. Pustylnik, M. Rubin-Zuzic, ' H. M. Thomas, ${ }^{1}$ (D) A. M. Lipaev, ${ }^{2}$ A. D. Usachev, V. I. Molotkov, ${ }^{2, a)}$ and M. H. Thoma ${ }^{3}$ (D)

\author{
AFFILIATIONS \\ ${ }^{1}$ Institut für Materialphysik im Weltraum, Deutsches Zentrum für Luft- und Raumfahrt e.V. (DLR), 82234 Wessling, Germany \\ 2Joint Institute for High Temperatures, Russian Academy of Sciences, 125412 Moscow, Russia \\ ${ }^{3}$ I. Physikalisches Institut, Justus-Liebig Universität, 35392 Gießen, Germany
}

\begin{abstract}
a) Note: Our very much admired and respected friend and colleague Vladimir Ivanovich Molotkov passed away unexpectedly on July 11, 2019, when this paper was finalized for submission.
\end{abstract}

\begin{abstract}
The charge of microparticles immersed in the dc discharge of the Plasmakristall-4 experimental facility has been estimated using the particle velocities from experiments performed on Earth and under microgravity conditions on the International Space Station. The theoretical model used for these estimates is based on the balance of the forces acting on a single particle in the discharge. The model takes into account the radial dependence of the discharge parameters and describes reasonably well the experimental measurements.
\end{abstract}

Published under license by AIP Publishing. https://doi.org/10.1063/1.5122861

\section{INTRODUCTION}

Micrometer sized particles immersed into a plasma form strongly coupled assemblies, which are the subject of active investigations in the field of complex (dusty) plasmas. Being relatively easy to resolve at a single particle level, such systems can be successfully used for modeling the kinetic properties in condensed matter physics. ${ }^{1,2}$ In order to better control and explain the behavior of the particle assemblies, it is necessary to have knowledge about the basic mechanisms of the plasmaparticle interaction, especially the charging process. Similar to the previous work, ${ }^{3}$ we estimate the charge of the microparticles drifting in the direct current (dc) discharge of the complex plasma laboratory, Plasmakristall-4 (PK-4). The facility was installed in the Columbus module of the International Space Station (ISS) in November 2014. ${ }^{4}$ It is an experimental laboratory with the possibility to provide a range of various experiments in the $\mathrm{dc}$ as well as radio frequency (rf) low temperature discharge including different manipulation techniques (e.g., laser manipulation and thermal and electric disturbances). Having an elongated shape of the plasma chamber, this set-up is particularly suitable for studying the flow-related phenomena in fluid complex plasmas at the kinetic level. Since the installation of PK-4 onboard the ISS, several scientific campaigns were performed with sets of different dedicated experiments (e.g., shear flows ${ }^{5}$ and low frequency waves ${ }^{6,7}$ ).

We concentrate on the analysis of a series of experiments with microparticles drifting in the plasma chamber tube. In a previously published work, ${ }^{3}$ it was observed that the particle drift velocities in the $\mathrm{dc}$ discharge of PK-4 under gravity conditions are always higher than those measured during parabolic flights in microgravity at identical experimental parameters. The developed analytical model provided the explanation of this phenomenon based on one of the main plasma-particle interaction mechanisms-the charging process. It was concluded that the observed difference in the particle drift velocities comes from the variation of particle positions in the discharge: under microgravity conditions, particles are levitating in the plasma bulk close to the discharge axis; in the ground-based laboratory, they are shifted in the vertical direction toward the chamber walls due to the force of gravity. Particle charge somewhat increases from the tube axis toward the periphery, and this explains the higher drift velocities observed in laboratory conditions. These results show the importance of the determination of the local distribution of the discharge parameters as well as the particle charge.

In this paper, we present the analysis of particle drift flows in the PK-4 tube from a series of experiments made in the groundbased laboratory and under microgravity conditions onboard the ISS. Using a theoretical model of particle charging, which takes into account the radial dependence of the discharge parameters within the discharge tube, we compare experimentally the measured particle velocities with those obtained using the model and estimate the microparticle charge. 


\section{EXPERIMENTAL PROCEDURE}

The experiments have been performed in PK-4 onboard the ISS as well as using the Scientific Reference Model 1 (SRM 1) of PK-4 built identical to the Flight Model in the ground based laboratory. The plasma is generated inside a U-shaped glass tube (horizontal part has a length of $35 \mathrm{~cm}$ ) with a radius of $1.5 \mathrm{~cm}$ by a dc discharge with high voltage $(\mathrm{HV})$ in the range of $0-3 \mathrm{kV}$, and a current of $0-3 \mathrm{~mA}$ is applied to the active electrode. The discharge tube on the ground is in the horizontal configuration. Complex plasma is formed by injecting spherical melamine-formaldehyde particles of different sizes into the discharge chamber.

Once particles are introduced into the plasma, they usually acquire a negative charge (due to highly mobile electrons) and drift from the cathode to the anode with a typical velocity of $1-10 \mathrm{~cm} / \mathrm{s}$. In laboratory conditions, the microparticle cloud is shifted downward [Fig. 1(a)], because a significant radial electric field is required to compensate for gravity. Under microgravity conditions, the microparticles are located in the vicinity of the discharge axis [Fig. 1(b)]. Here, they form clouds of several layers with a distance between the microparticles (depending on the discharge parameters and microparticle size) ranging from 100 to $300 \mu \mathrm{m}$. The microparticle suspensions are illuminated by a laser diode with a maximum power of $150 \mathrm{~mW}$ and a wavelength of $532 \mathrm{~nm}$. The light scattered by the microparticles is recorded using two video cameras, which allow us to observe a field of view (FoV) of $44 \times 17 \mathrm{~mm}^{2}$ with a maximum resolution of approximately $14 \mu \mathrm{m}$ and a frame rate of $35 \mathrm{fps}$.

Besides dc discharge, the PK-4 facility has the possibility to ignite an inductively coupled rf discharge with $81.36 \mathrm{MHz}$ and a maximal forward power of $5 \mathrm{~W}$ using two rf coils: one coil has a fixed position and the other can be moved along the discharge tube. A more detailed description of the PK-4 apparatus is presented in Ref. 4.

One of the experiments performed on ISS was dedicated to estimate the velocities of the particles drifting in the dc discharge tube. In order to have better statistics in velocity measurements and control over the microparticle suspension, a combined microparticle transport and trapping technique ( $\mathrm{dc}$ discharge transport and rf discharge trapping) was applied (see Ref. 4, Sec. II H).

In this method, the injected negatively charged microparticles were first transported to the rf discharge of the movable rf coil by the longitudinal electric field of the dc discharge. Then, the dc plasma was switched off and microparticles were stored in the rf discharge. Afterward, a polarity-switched plasma was ignited with a $50 \%$ duty cycle and a frequency of $500 \mathrm{~Hz}$ and the rf discharge was switched off. Next, a dc discharge was applied first with a negative current and the microparticles were drifting for a certain time in one direction. Later, the polarity was reversed and the microparticles were drifting in the opposite direction. This allowed us to get more statistics on microparticle drift velocity.

The experimental procedure was similar to that described previously. ${ }^{6}$ But the experiments were performed in a broader range of parameters on board the ISS as well as in the ground laboratory. Neon and argon gases were used in the pressure range of $20-100 \mathrm{~Pa}$. Monodisperse plastic (melamine formaldehyde) microparticles of three different sizes $(1.3,2.5$, and $3.4 \mu \mathrm{m}$ in diameter) were injected into a discharge. There was a small gas leak of about $0.1 \mathrm{sccm}$ observed during the experiments as mentioned in Ref. 6. The dc discharge was switched on with different values of the discharge current $(0.5-1.5 \mathrm{~mA})$. The duration time for the measurement procedure was between 1 and $3 \mathrm{~s}$ in each direction depending on the discharge current (the higher the discharge current, the slower the particles are moving). The rf discharge was ignited with constant powers of $1 \mathrm{~W}$ for argon and $0.4 \mathrm{~W}$ for neon gas. The experimental procedure on the ISS was similar to that performed in the ground laboratory. On the ISS, only particles with a diameter of $3.4 \mu \mathrm{m}$ were used for this experiment.

Experimentally, the particle velocities were estimated in two ways for comparison. In the first method, the velocity was determined from the time of flight of the suspension through the FoV [Figs. 2(a) and 2(b)]. Another method was relying on the slope in the space-time diagram of the microparticle motion [Fig. 2(c)]. If one has sequential images, where moving particles are seen whose coordinates cannot be obtained (e.g., since they overlap), it is possible to gain additional dynamical information from the corresponding "space time diagrams." The main idea is to measure the mean brightness in columns and rows as a function of time (image number). From the obtained images, e.g., the mean velocity of the particle cloud can be measured from the slope seen in such a space time diagram. Here, one needs to take into account the fact that the additional gas velocity due to the gas flow leak, as it has been mentioned in Ref. 6, was in the range of 0.6 to $1 \mathrm{~cm} / \mathrm{s}$ depending on the gas pressure.

In order to characterize the discharge, Langmuir probe measurements were performed in the particle-free plasma on the axis of the
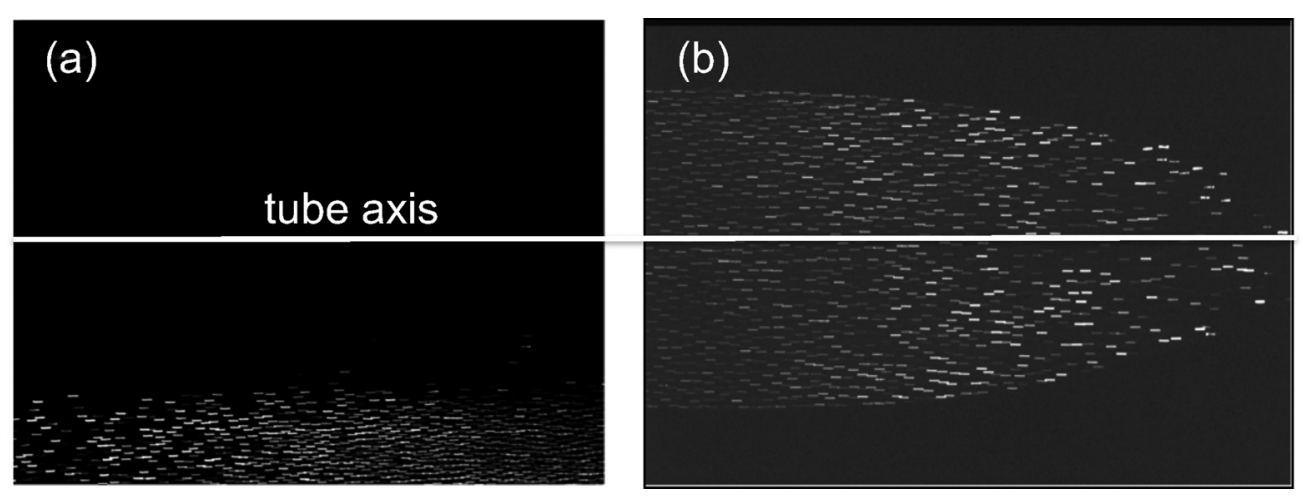

FIG. 1. The examples of the drift flow of $3.4 \mu \mathrm{m}$ diameter particles in argon dc discharge of PK-4 at a pressure of $100 \mathrm{~Pa}$ and a discharge current of $1 \mathrm{~mA}$ in laboratory conditions (a) and under microgravity conditions on ISS (b). The pictures are taken with a frame rate of $35 \mathrm{fps}$ and an exposure time of $28 \mathrm{~ms}$. 

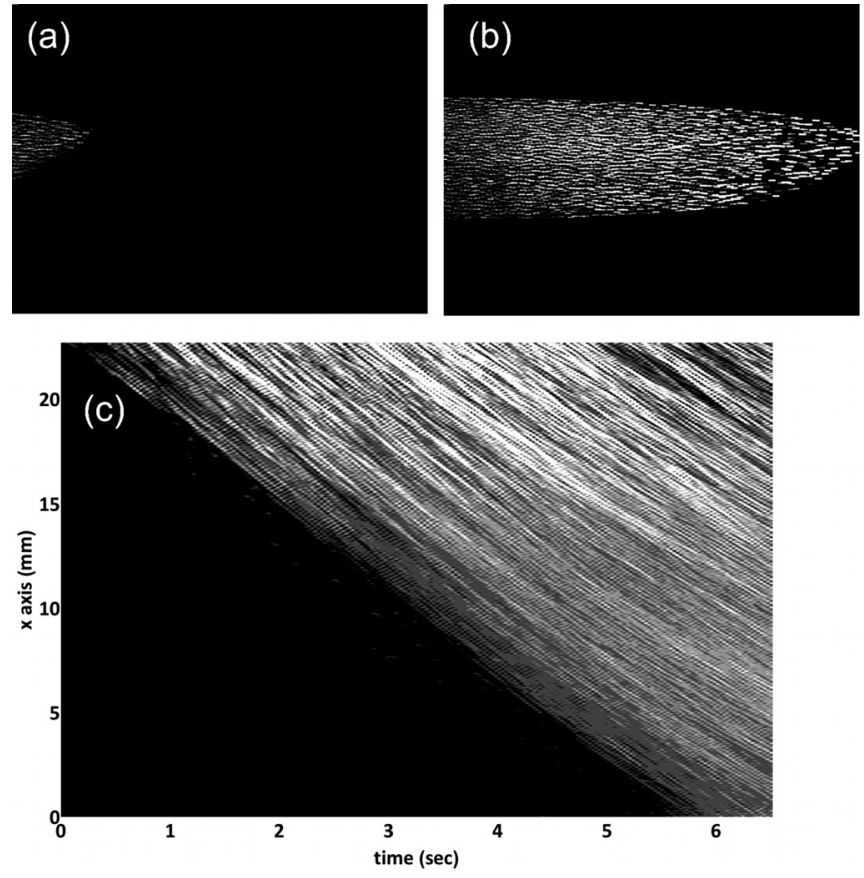

FIG. 2. The upper pictures (a) and (b) show the front of the particle cloud entering and leaving the FoV (particles flow from left to right). The lower picture (c) shows the space-time diagram for the sequence of frames (140 frames). The pictures are taken from the experiment done on the ISS with the $3.4 \mu \mathrm{m}$ diameter particles in neon dc discharge, a pressure of $60 \mathrm{~Pa}$, and a discharge current of $1 \mathrm{~mA}$.

discharge tube at different experimental conditions in a ground setup identical to the present PK-4 models. ${ }^{8}$ The discharge parameters such as electron density, electron temperature, and electric field values measured in the pressure range of $20-100 \mathrm{~Pa}$ in neon and argon gases at a discharge current of $1 \mathrm{~mA}$ are presented in Figs. 3 and 4, respectively.

These measurements showed that for neon plasma, the electron density increases with pressure, the electron temperature slightly decreases, and the electric field remains almost the same in the chosen pressure range. At the same time, for argon plasma, the measurements showed different dependences of the plasma parameters on pressure: the electron temperature and electric field increase with pressure. The electron density shows the same dependence on the pressure as for neon. These measured plasma parameters or their interpolations (if the experimental conditions were different from the conditions in Ref. 8) were used in order to estimate the particle charge values in the dc discharge of PK-4.

\section{THEORETICAL MODEL}

The theoretical model used in this work is based on the force balance condition. For simplicity, we consider a single particle and neglect possible effects of the particle component on the discharge parameters. As has been previously shown ${ }^{9}$ even in a relatively dense cloud, the electric force is dominant. Under microgravity conditions, only the longitudinal direction is considered, assuming that the test particle is located near the discharge axis. The main forces include the electric force, the ion drag force, and the neutral drag forces ${ }^{1,10}$ (the electron drag force can also be important for relatively low electron

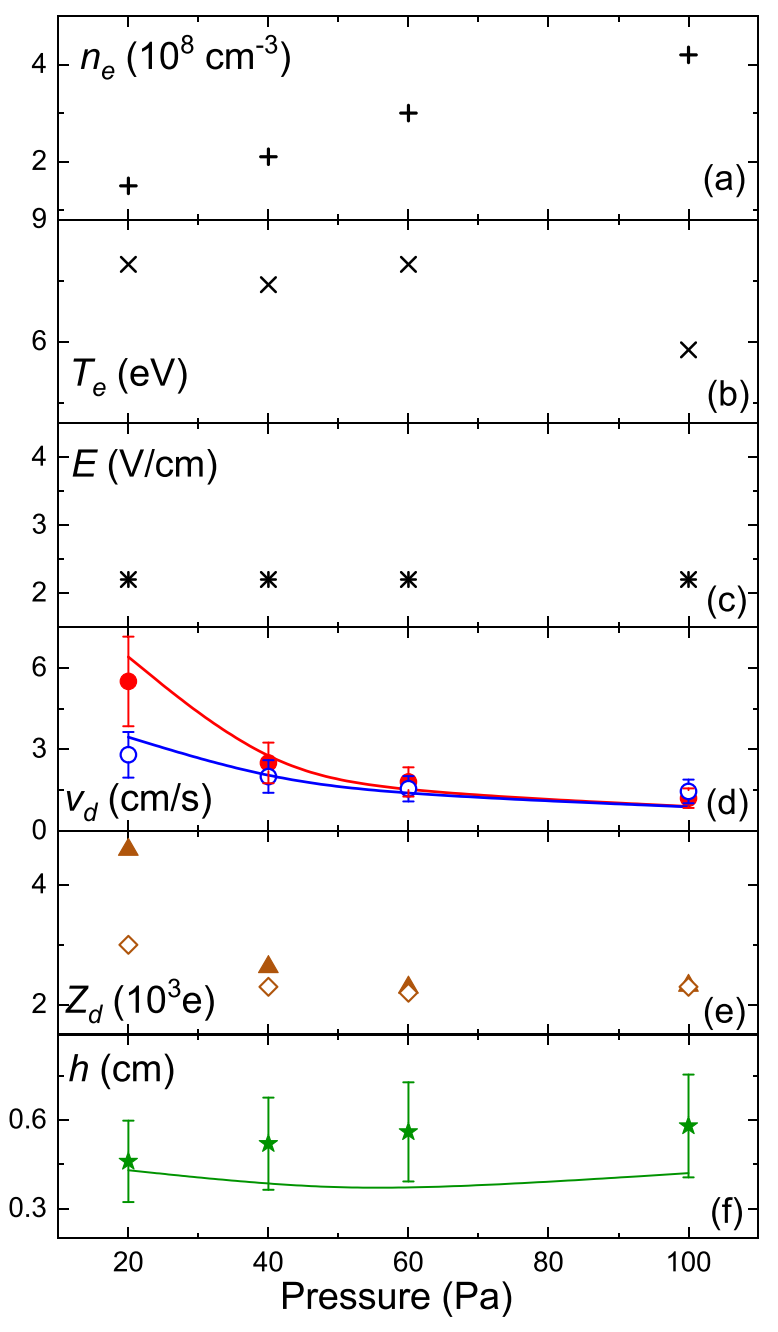

FIG. 3. Pressure dependence on the main plasma parameters (electron density $n_{e}$, electron temperature $T_{e}$, and electric field $E$ ), experimentally measured (circles) and theoretically estimated (curves) microparticle drift velocities $V_{d}$ for ground (red color) and microgravity (blue color) conditions with experimental uncertainties, theoretically estimated microparticle charge $Z_{d}$ for ground (triangle) and microgravity (diamond) conditions, distance of the upper microparticle layer from the center of the tube $h$ measured on ground (stars) and theoretically estimated (curve). All the values are presented for the case of neon gas, $1 \mathrm{~mA}$ discharge current, and $3.4 \mu \mathrm{m}$ diameter microparticles.

temperatures, ${ }^{11}$ but not for the conditions investigated here). The microparticle drift velocity can be simply estimated from the force balance $F_{e l}^{\|}+F_{i}^{\|}+F_{n}=0$, like in Ref. 3. Here, $F_{e l}^{\|}$is the horizontal component of the electric force, $F_{i}^{\|}$is the horizontal component of the ion drag force, and $F_{n}$ is the neutral drag force. In ground laboratory experiments, the particles levitating in the wall region experience a longitudinal as well as radial component of the electric field, and hence, the force balance here includes the radial component of the electric and ion drag forces as well as the gravity force $F_{e l}^{\perp}+F_{i}^{\perp}+F_{g}=0$, where $F_{g}$ is the force of gravity. The force evaluation is supplemented by the appropriate charging model. The charge is calculated from the 


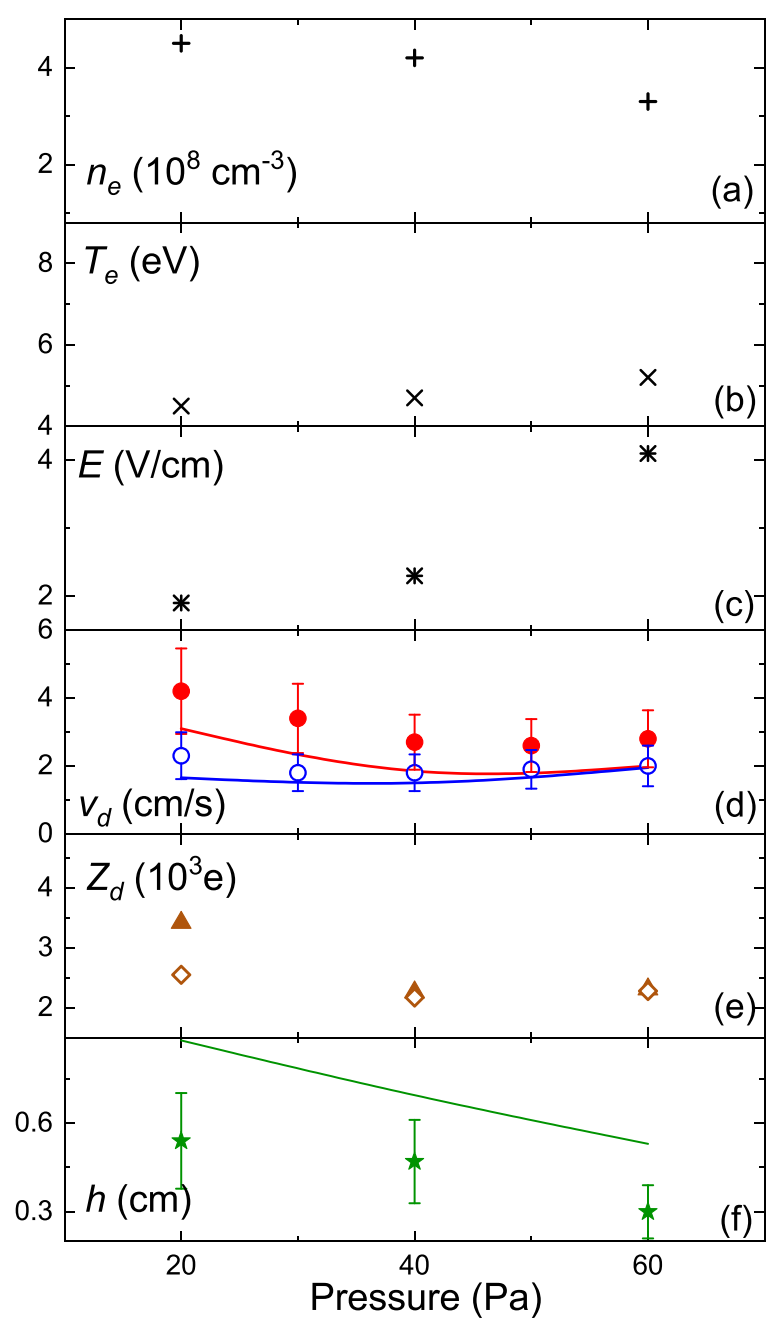

FIG. 4. The same as in Fig. 3, but for argon gas.

balance of electron and ion fluxes to the particle surface taking into account the effect of ion-neutral collisions ${ }^{12-16}$ and ionization enhancement of the ion flux to the particle surface. ${ }^{17}$

The model used is very similar to that described in Ref. 3, which allows us to omit the details. The following improvements and updates have been implemented.

The radial dependence of the plasma density and electric field has been explicitly introduced using the simplest approximation, ${ }^{18}$

$$
n_{i} \simeq n_{e} \simeq n_{0} J_{0}(2.4 r / R)
$$

and

$$
E_{\perp}=2.4 \frac{T_{e}}{e R} \frac{J_{1}(2.4 r / R)}{J_{0}(2.4 r / R)},
$$

where $n_{0}$ is the ion and electron densities on the tube axis, $R$ is the tube radius, $T_{e}$ is the electron temperature, $e$ is the elementary charge, and $J_{0,1}(x)$ are Bessel functions of the first kind. Electron impact ionization rates were taken from Ref. 19. Here, one needs to note that the overestimation of the ionization effect due to non-Maxwellian electron energy distribution can lead to the underestimation of the microparticle charge. ${ }^{20}$

According to some recent results, ${ }^{21,22}$ the electron absorption coefficient for collisions with the particle surface was allowed to be different from unity. We have considered two cases, when it is equal to 1 and 0.5 , respectively. The estimations for these both cases do not differ much, and therefore, further we present our results for the case where the electron absorption coefficient is equal unity. Collisional enhancement of the ion drag force has been modeled using a correction factor proposed previously. ${ }^{23}$ We have also used a simple approximation for the effective ion-neutral collision frequency. ${ }^{24}$

\section{RESULTS}

Figures 3 and 4 illustrate the comparison between experimental and theoretical results. Figure 3 shows the dependence of the measured plasma parameters (electron density $n_{e}$, electron temperature $T_{e}$, and electric field $E$ ) on the gas pressure. In addition, the experimental and theoretical drift velocities $v_{d}$, theoretically estimated microparticle charge $Z_{d}$ on ground and under microgravity conditions, and the distance of the upper microparticle layer from the center of the tube $h$ measured on ground and theoretically estimated are presented here. The data shown are for the $3.4 \mu \mathrm{m}$ diameter particles in neon gas at a discharge current of $1 \mathrm{~mA}$. The experimental uncertainties are shown for the microparticle drift velocity $v_{d}$ and for the distance of the upper microparticle layer from the center of the tube $h$ and come from the determination of the microparticle positions. Here, one can see that by increasing the discharge pressure, the microparticles become slower because of the neutral friction. The theoretically estimated microparticle drift velocity demonstrates that the chosen analytical model reproduces relatively well the experimental measurements.

In addition, the data of experiments performed on ISS confirm previous observations from parabolic flights ${ }^{3}$ that microparticles in ground-based experiments move faster than those under microgravity conditions. The difference is more pronounced for the lower pressure range $(20-30 \mathrm{~Pa})$. The theoretical curves are in agreement with this tendency. The measured drift velocities in the case of neon gas for $3.4 \mu \mathrm{m}$ diameter particles quantitatively agree well with those measured during parabolic flights. ${ }^{3}$

Figure 4 shows similar dependencies for argon gas. Here, the microparticle velocity does not exhibit a monotonic decrease in the pressure as in the neon case in Fig. 3. Instead, in the region of moderate pressures $(60 \mathrm{~Pa})$, the microparticle velocity somewhat increases with pressure. It can be explained by the nonmonotonic dependence of the plasma parameters on pressure, namely, electron temperature $T_{e}$ and electric field $E$. Lower values of $T_{e}$ in the case of argon compared to neon lead to a lower particle charge and, hence, lower microparticle velocities. On the other hand, the electric field $E$ is almost constant in the chosen pressure range. The results from the theoretical model are consistent with this behavior.

The theoretically estimated charge of the $3.4 \mu \mathrm{m}$ diameter particles presented for argon and neon in Figs. 3 and 4 also shows a different dependence on the pressure for argon and neon gases. In the case of argon plasma, the charge is estimated to be lower than that in neon plasma. This is mainly related to the higher electron temperature in neon gas. 
The model presented above includes the radial distribution of the discharge parameters within the discharge tube. This allows us to compare the distance of the upper microparticle layer from the center of the tube $h$ estimated from the experimental measurements on ground with that of a single particle obtained from theoretical calculations performed here.

The comparison in Figs. 3 and 4 for the case of neon and argon discharges shows some discrepancy between theory and experiments, although it shows better agreement in the case of neon gas. The reason for this discrepancy could be the assumption of the single particle model in the theoretical consideration. Although in the experiments, we tried to keep the particle density as low as possible, we could not always achieve it. The estimations of the microparticle influence on the electric field, as it has been discussed in Ref. 25, have shown that the electric field during the ISS experiments, e.g., for the conditions presented in Fig. 2 (neon dc discharge, $60 \mathrm{~Pa}$ pressure, and $1 \mathrm{~mA}$ discharge current) is about $30 \%$ higher than that measured by the Langmuir probe in the particle-free discharge (see Figs. 3 and 4).

In addition, the number of particles could vary from one experiment to another. Finally, it cannot be excluded that the radial distribution of plasma density and electric field does not follow closely the simple theoretical approximation used in this work. However, a recent analysis of collective excitations ${ }^{7}$ for the wave experiments performed in PK-4, which includes the charge estimation from our model and assumes the so-called Havnes parameter approximately equal to unity, has not shown large inconsistency with the experiment.

Another possible reason for the deviation between the theory and experimental measurements could be the change in the microparticle mass and shape during the exposure in the plasma discharge as it has been reported in several works. ${ }^{26-30}$ Since in our case, the time of the microparticle levitation in the discharge corresponds to a minute time scale, we do not expect this effect to be very important.

In Fig. 5, we have plotted the theoretically estimated reduced (normalized) charge $z=Z_{d} e^{2} / a T_{e}$, where $e$ is the elementary electron charge and $a$ is the radius of the $3.4 \mu \mathrm{m}$ diameter particles, vs pressure for argon plasma at $I=1.0 \mathrm{~mA}$ and neon plasma at $I=0.5,1.0$, and $1.5 \mathrm{~mA}$. The charge calculation refers to the tube axis region. The

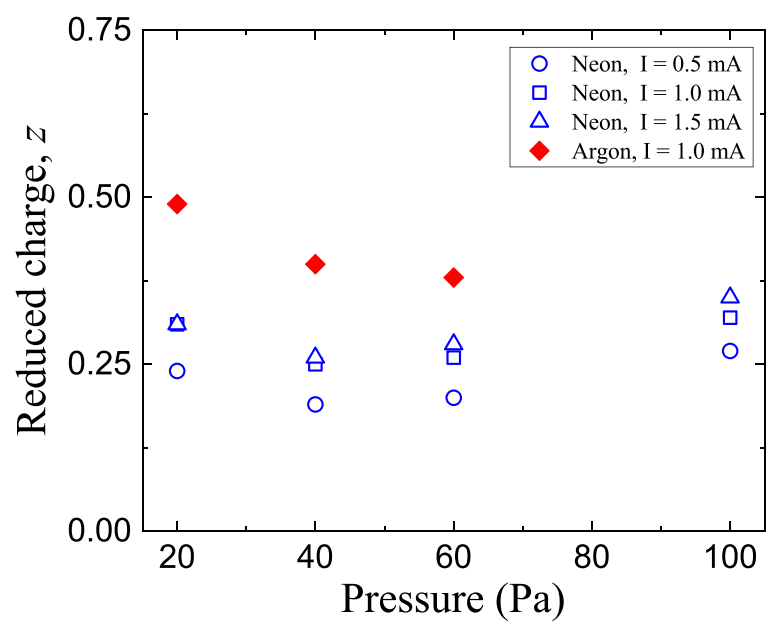

FIG. 5. Reduced charge of $3.4 \mu \mathrm{m}$ diameter particles at the discharge tube axis vs pressure for neon and argon gases at several discharge current values. reduced charge is essentially the particle surface potential in units of $T_{e} / e$. The reduced charge is higher for argon plasma, mainly due to the lower electron-to-ion mass ratio. ${ }^{31}$ It does not vary much neither with pressure nor with discharge current in the investigated parameter regime. The averaged values are $z=0.3 \pm 0.1$ for neon and $z=0.4 \pm 0.1$ for argon. Note that this is about one order of magnitude smaller than the orbit motion limited theory would yield. ${ }^{31}$ Since the reduced charge does not depend strongly on the particle size, the values shown should be generally representative for PK-4 experiments under microgravity conditions.

\section{CONCLUSION}

In this work, the microparticle drift velocities from the analytical model, which takes into account the local distribution of the discharge parameters, were compared with those from the experiments performed in a laboratory set-up on the ground and under microgravity conditions on the ISS. The experiments were carried out in a broad range of discharge parameters (pressure and discharge current) and in two different gases: argon and neon. Both the experimentally measured and theoretically estimated particle velocities and charges show different behavior on the pressure for argon and neon plasmas. Also, the quantitative values of charge differ for these two gases. The reported values of particle charge can be used as reference values for other experiments in the PK-4 laboratory.

\section{ACKNOWLEDGMENTS}

All the authors greatly acknowledge the joint ESA-Roscosmos "Experiment Plasmakristall-4" on board the International Space Station. This work was partially supported by DLR Grant Nos. 50WM1441 and 50WM1442. We also thank Dr. Yaroshenko for comments and critical reading of this manuscript.

\section{REFERENCES}

${ }^{1}$ V. E. Fortov, A. V. Ivlev, S. A. Khrapak, A. G. Khrapak, and G. E. Morfill, Phys. Rep. 421, 1-103 (2005).

${ }^{2}$ G. E. Morfill and A. V. Ivlev, Rev. Mod. Phys. 81, 1353-1404 (2009).

${ }^{3}$ S. A. Khrapak, M. H. Thoma, M. Chaudhuri, G. E. Morfill, A. V. Zobnin, A. D. Usachev, O. F. Petrov, and V. E. Fortov, Phys. Rev. E 87, 063109 (2013).

${ }^{4}$ M. Y. Pustylnik, M. A. Fink, V. Nosenko, T. Antonova, T. Hagl, H. M. Thomas, A. V. Zobnin, A. M. Lipaev, A. D. Usachev, V. I. Molotkov et al., Rev. Sci. Instrum. 87, 093505 (2016).

${ }^{5}$ M. Weber, M. Fink, V. Fortov, A. Lipaev, V. Molotkov, G. Morfill, O. Petrov, M. Pustylnik, M. Thoma, H. Thomas, A. Usachev, and C. Raeth, Opt. Express 24, 7987-8012 (2016).

${ }^{6}$ S. Jaiswal, M. Pustylnik, S. Zhdanov, H. M. Thomas, A. M. Lipaev, A. D. Usachev, V. I. Molotkov, V. E. Fortov, M. H. Thoma, and O. V. Novitskii, Phys. Plasmas 25, 083705 (2018).

${ }^{7}$ V. V. Yaroshenko, S. A. Khrapak, M. Y. Pustylnik, H. M. Thomas, S. Jaiswal, A. M. Lipaev, A. D. Usachev, O. F. Petrov, and V. E. Fortov, Phys. Plasmas 26, 053702 (2019).

${ }^{8}$ V. E. Fortov, G. E. Morfill, O. Petrov, M. H. Thoma, A. D. Usachev, H. Höfner, A. V. Zobnin, M. Kretschmer, S. Ratynskaya, M. A. Fink, K. Tarantik, Y. Gerasimov, and V. Esenkov, Plasma Phys. Controlled Fusion 47, B537 (2005).

${ }^{9}$ V. V. Yaroshenko, S. A. Khrapak, and G. E. Morfill, Phys. Plasmas 20, 043703 (2013).

${ }^{10}$ V. E. Fortov, A. G. Khrapak, S. A. Khrapak, V. I. Molotkov, and O. F. Petrov, Phys.-Usp. 47, 447-492 (2004).

${ }^{11}$ S. A. Khrapak and G. E. Morfill, Phys. Rev. E 69, 066411 (2004).

${ }^{12}$ A. V. Zobnin, A. P. Nefedov, V. A. Sinel'shchikov, and V. E. Fortov, JETP 91, 483-487 (2000). 
${ }^{13}$ M. Lampe, R. Goswami, Z. Sternovsky, S. Robertson, V. Gavrishchaka, G. Ganguli, and G. Joyce, Phys. Plasmas 10, 1500-1513 (2003).

${ }^{14}$ S. Ratynskaia, S. Khrapak, A. Zobnin, M. H. Thoma, M. Kretschmer, A. Usachev, V. Yaroshenko, R. A. Quinn, G. E. Morfill, O. Petrov, and V. Fortov, Phys. Rev. Lett. 93, 085001 (2004).

${ }^{15}$ S. A. Khrapak, S. V. Ratynskaia, A. V. Zobnin, A. D. Usachev, V. V. Yaroshenko, M. H. Thoma, M. Kretschmer, H. Höfner, G. E. Morfill, O. F. Petrov, and V. E. Fortov, Phys. Rev. E 72, 016406 (2005).

${ }^{16}$ S. Khrapak and G. Morfill, Contrib. Plasma Phys. 49, 148 (2009).

${ }^{17}$ S. A. Khrapak and G. E. Morfill, Phys. Plasmas 19, 024510 (2012).

${ }^{18}$ Y. P. Raizer, Gas Discharge Physics (Springer, 2011).

${ }^{19}$ M. A. Lennon, K. L. Bell, H. B. Gilbody, J. G. Hughes, A. E. Kingston, M. J. Murray, and F. J. Smith, J. Phys. Chem. Ref. Data 17, 1285-1363 (1988).

${ }^{20}$ V. A. Godyak, R. B. Piejak, and B. M. Alexandrovich, Plasma Sources Sci. Technol. 11, 525-543 (2002).

${ }^{21}$ F. X. Bronold and H. Fehske, Phys. Rev. Lett. 115, 225001 (2015).

${ }^{22}$ A. V. Zobnin, A. D. Usachev, O. F. Petrov, V. E. Fortov, M. H. Thoma, and M. A. Fink, Phys. Plasmas 25, 033702 (2018)
${ }^{23}$ I. H. Hutchinson and C. B. Haakonsen, Phys. Plasmas 20, 083701 (2013).

${ }^{24}$ S. Khrapak, J. Plasma Phys. 79, 1123-1124 (2013).

${ }^{25}$ A. D. Usachev, A. V. Zobnin, O. F. Petrov, V. E. Fortov, M. H. Thoma, M. Y. Pustylnik, M. A. Fink, and G. E. Morfill, Plasma Sources Sci. Technol. 25, 035009 (2016).

${ }^{26}$ J. Pavlů, A. Velyhan, I. Richterová, Z. Němeček, J. Šafránková, I. Čermák, and P. Žilavý, IEEE Trans. Plasma Sci. 32, 704 (2004).

${ }^{27}$ J. Carstensen, H. Jung, F. Greiner, and A. Piel, Phys. Plasmas 18, 033701 (2011).

${ }^{28}$ C. Killer, F. Greiner, S. Groth, B. Tadsen, and A. Melzer, Plasma Sources Sci. Technol. 25, 055004 (2016).

${ }^{29}$ M. Vysinka, L. Nouzak, J. Pavlu, Z. Nemecek, J. Safrankova, and I. Richterova, IEEE Trans. Plasma Sci. 46, 709 (2018).

${ }^{30}$ V. Yu. Karasev, E. S. Dzlieva, S. I. Pavlov, O. V. Matvievskaya, V. A. Polischuk, M. A. Ermolenko, A. I. Eikhval'd, and A. P. Gorbenko, Contrib. Plasma Phys. 59, e201800145 (2019).

${ }^{31}$ S. A. Khrapak, A. V. Ivlev, and G. Morfill, Phys. Rev. E 64, 046403 (2001). 